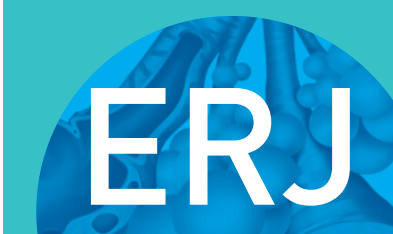

open research

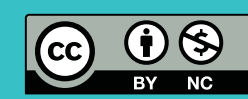

\section{Lung function and disease severity in cystic fibrosis patients heterozygous for p.Arg117His}

\author{
Michal Shteinberg ${ }^{1,2,3}$, Damian G. Downey ${ }^{1,3}$, Diane Beattie ${ }^{1}, J^{2}$ ohn McCaughan ${ }^{4}$, \\ Alastair Reid ${ }^{4}$, Nili Stein ${ }^{2}$ and J. Stuart Elborn ${ }^{1,3,5}$
}

Affiliations: ${ }^{1}$ Northern Ireland Regional Adult Cystic Fibrosis Centre, Belfast Health and Social Care Trust, Belfast, UK. ${ }^{2}$ Pulmonology and CF Center, Carmel Medical Center and Rappaport Faculty of Medicine, Haifa, Israel. ${ }^{3}$ Queen's University of Belfast, Belfast, UK. ${ }^{4}$ Northern Ireland Regional Paediatric Cystic Fibrosis Centre, Royal Belfast Hospital for Sick Children, Belfast Health and Social Care Trust, Belfast, UK. ${ }^{5}$ mperial College and Royal Brompton Hospital, London, UK.

Correspondence: J. Stuart Elborn, Centre of Infection and Immunity, Health Sciences Building, 97 Lisburn Road, Belfast, BT9 7BL, UK. E-mail: s.elborndqub.ac.uk

ABSTRACT Expression of p.Arg117His cystic fibrosis (CF) transmembrane conductance regulator is influenced by a polythymidine (poly-T) tract and a thymidine-guanine (TG) repeat on intron 9, which vary in length and affect exon 10 skipping.

We compared clinical characteristics and the rate of progression of lung disease of CF patients carrying the p.Arg117His mutation with different intron 9 varying sequences (poly-T) and mutation classes in trans.

Data were collected from patients in Northern Ireland, UK, including diagnostic features, sweat chloride, nutritional status, sputum microbiology, CF-related complications and lung function. Poly-T and TG repeats were determined by PCR. Forced expiratory volume in $1 \mathrm{~s}(\mathrm{FEV} 1)$ decline was determined from linear regression of FEV1 measurements of patients over time.

We identified 62 patients with p.Arg117His, 55 with a class I/II mutation in trans and six with p.Arg117His/p.Gly551Asp. 42 patients had 5T and 13 had 7T. All patients had 12 TG repeats. Patients with p.Arg117His-5T had greater lung function decline, sweat chloride concentrations, pancreatic insufficiency and prevalence of Pseudomonas aeruginosa infection compared with patients with p.Arg117His-7T.

Lung function decline and disease severity in p.Arg117His is determined by the poly-T tract length and identity of the mutation in trans. Patients with p.Arg117His-5T and a second class I/II mutation have a severity similar to p.Phe508del homozygous patients, although lung function decline is delayed to an older age. There may be linkage disequilibrium between p.Arg117His and 12 TG repeats.

@ERSpublications

p.Arg117His CFTR with 5T repeats is associated with accelerated lung function decline compared with p.Arg117His-7T http://ow.ly/yAdS308q3dn

Cite this article as: Shteinberg M, Downey DG, Beattie D, et al. Lung function and disease severity in cystic fibrosis patients heterozygous for p.Arg117His. ERJ Open Res 2017; 3: 00056-2016 [https:// doi.org/10.1183/23120541.00056-2016].

Received: May 102016 | Accepted after revision: Jan 192017

Conflict of interest: Disclosures can be found alongside this article at openres.ersjournals.com

Copyright $\odot$ ERS 2017. This article is open access and distributed under the terms of the Creative Commons Attribution Non-Commercial Licence 4.0. 


\section{Introduction}

Cystic fibrosis (CF) is caused by mutations in the gene encoding the CF transmembrane regulator (CFTR) protein, a chloride channel located in the luminal membrane of epithelial cells. Over 2000 CFTR mutations have been found, and are classified into five classes (I-V) according to the affected step in protein translation and function [1]. Briefly, these mutation classes are: class I, impaired protein syntheses; class II, protein degradation; class III, altered function, e.g. blocked CFTR channel (altered gating); class IV, reduced conductance of the CFTR; and class V, reduced amount of CFTR. CF genotypes which result in partial functioning CFTR (classes IV and V) are usually associated with late diagnosis and a milder phenotype [2].

The p.Arg117His (formerly R117H) mutation results in a substitution of histidine for arginine at position 117 of the protein. The CFTR channel generated from p.Arg117His has reduced chloride conductance and altered channel gating [3]. Individuals homozygous for the p.Arg117His mutation are rarely described and when identified have very limited manifestations of CFTR dysfunction, typically male infertility with little or no sino-pulmonary disease [4-6]. Compound heterozygotes, carrying one severe CF-causing mutation and p.Arg117His, have a less severe phenotype than individuals with class I-III mutations; some have no disease at the time of identification. This has been estimated by calculation of the penetrance of CF among individuals expected to carry the p.Arg117His/p.Phe508del mutations [7]. This has led to some debate as to whether the p.Arg117His mutation should be included in newborn screening panels for CF [7, 8]. Disease severity may also be influenced by the mutation in trans with p.Arg117His. COMER et al. [9] found that patients with p.Arg117His and a class I/II mutation have a more severe phenotype than patients with $p$.Arg117His and a class III mutation.

The variable phenotypes of people with $\mathrm{CF}$ and the p.Arg117His mutation can in part be explained by alternative splicing. Intron 9 (IVS9; formerly exon 8 by previous nomenclature) of the CFTR gene contains a variable sequence of five, seven or nine thymidine bases ("polythymidine (poly-T) repeats"), which are immediately adjacent to a splicing site on exon 10 (formerly exon 9). All poly-T variants result in exon 10 skipping. The $5 \mathrm{~T}$ allele is associated with the least effective exon 10 retention, resulting in high skipping levels of exon 10 (exon 10-). The 9T allele is associated with the highest levels of exon 10 retention, Hence, the proportion of "exon 10-" mRNA gradually decreases from 9T to 7T and 5T individuals, both $\mathrm{CF}$ and non-CF [10]. When found in trans with another disease-causing mutation, the poly-T tract variable sequence on intron 9 (IVS9T) can be associated with congenital bilateral absence of the vas deferens $[11,12]$ or no clinical consequences. However, some studies report patients with significant sino-pulmonary disease and a genotype of 5T in trans with a CF-causing mutation [13-15]. More commonly, when IVS9-5T is in cis with a mutated allele, such as p.Arg117His, and accompanied by a second CF-causing mutation, it may cause CF [16].

CF patients with the combination p.Phe508del/p.Arg117His-5T may have severe lung disease. MAssie et al. [16] described 41 Australian CF patients with p.Phe508del/p.Arg117His and known poly-T status. p.Phe508del/p.Arg117His-5T patients had lung disease consistent with CF, while p.Phe508del/ p.Arg117His-7T patients did not. Lung function was not significantly different between 5T and 7T patients; however, few patients had lung function data and lung function decline by age group was not assessed.

A second genetic variation that may influence protein levels is a sequence of thymidine-guanine (TG) dinucleotide repeats of varying length. This sequence lies immediately before the poly-T tract in intron 9 and may consist of 11,12 or 13 repeats [17-19]. When associated with 5T poly-T repeats, longer (12 or 13) repeats are associated with less effective splicing and lower protein product, and a higher disease penetrance [20].

The adult and paediatric CF centres care for all patients with CF in Northern Ireland, UK. It was previously found that $14.6 \%$ of these patients carry the p.Arg117His mutation [9] with varying degrees of disease severity. The aim of this study was to determine whether IVS9-5T is associated with greater decline in forced expiratory volume in $1 \mathrm{~s}(\mathrm{FEV} 1)$ than IVS9-7T CF in patients carrying a p.Arg117His mutation. Secondary objectives were to determine the decline in FEV1 of p.Arg117His patients compared with p.Phe508del homozygous patients and a small number of patients who were p.Gly551Asp/p.Arg117His heterozygotes.

\section{Methods}

We identified all patients in Northern Ireland carrying p.Arg117His and p.Phe508del homozygous through our CF patient registry. Ethics approval for data analysis from the registry was given (reference 07/Q0104/2). Retrospective data included diagnostic features, CFTR mutations, sweat chloride, nutritional status, CF-related complications and lung function. For patients with $p$.Arg117His who participated in a clinical trial with ivacaftor, all data collected were prior to study inclusion. IVS9T length and TG repeats were determined by PCR as described elsewhere [21] using patients' stored blood samples. This method is based on a single-step PCR, making use of an allele-specific reverse primer matching the 5T allele plus one additional nucleotide at the $3^{\prime}$ end. Accordingly, the complete 5T stretch is encapsulated within the primer sequence which prevents binding and amplification to the $7 \mathrm{~T}$ or $9 \mathrm{~T}$ alleles. 
Statistical analysis was performed using IBM SPSS Statistics version 21 (IBM, Armonk, NY, USA). Continuous variables are presented as mean, median and standard deviation. Categorical variables are presented as percentages. Continuous variables were compared using the independent $\mathrm{t}$-test or Mann-Whitney test, as appropriate. Categorical variables were compared using the Chi-squared test. The linear mixed model was used to examine if there were group differences in FEV 1 values with age. Logistic regression was used to compare differences in Pseudomonas aeruginosa prevalence between the groups adjusted for age. $\mathrm{p}$-values $<0.05$ were considered statistically significant.

\section{Results}

\section{Patient demographics and genetic variation}

In total, 62 patients with p.Arg117His were included in the analysis. Of these, 55 had p.Arg117His in trans with a class I or II mutation (figure 1). Six patients were compound heterozygotes for p.Arg117His/ p.Gly551Asp. No patients homozygous for p.Arg117His or compound heterozygotes for p.Arg117His with a class IV or $\mathrm{V}$ mutation were identified.

IVS9T status was determined for 54 out of 61 p.Arg117His patients. Of these, 42 patients had 5T and 12 patients had 7T. Determination of TG repeats was attempted for all stored DNA samples. However, TG repeats could not be reliably determined by the PCR method for the patients with 7T. In total, 39 patients with $5 \mathrm{~T}$ were analysed and all had $12 \mathrm{TG}$ repeats (figure 1).

\section{Clinical characteristics of different genetic variants of patients with the p.Arg117His mutation compared with p.Phe508del homozygotes}

To determine whether different genotypes influence clinical outcomes of severity, we grouped CF patients into the following four groups: 1) 39 patients with p.Arg117His and IVS9-5T, along with a second class I or II mutation ("5T"); 2) 10 patients with p.Arg117His and IVS9-7T, along with a second class I or II mutation ("7T"); 3) six patients with p.Arg117His and p.Gly551Asp (5T and 7T were grouped together due to the small number of patients in this group); and 4) 191 patients homozygous for p.Phe508del.

\section{Lung function}

Spirometry values, determined at every clinic visit and several times during hospitalisation for pulmonary exacerbations, were extracted from the patients' files. To determine lung function decline over time, we calculated median values of FEV1 \% pred over a calendar year for every patient. We used the linear mixed model to calculate $\mathrm{FEV}_{1} \%$ pred decline from the median FEV1 values for patients in each of the four groups (figure 2). Patients' ages in the different patient groups were not statistically different, and FEV1 decline with age was not different between younger and older patients. FEV1 \% pred decline per year was:

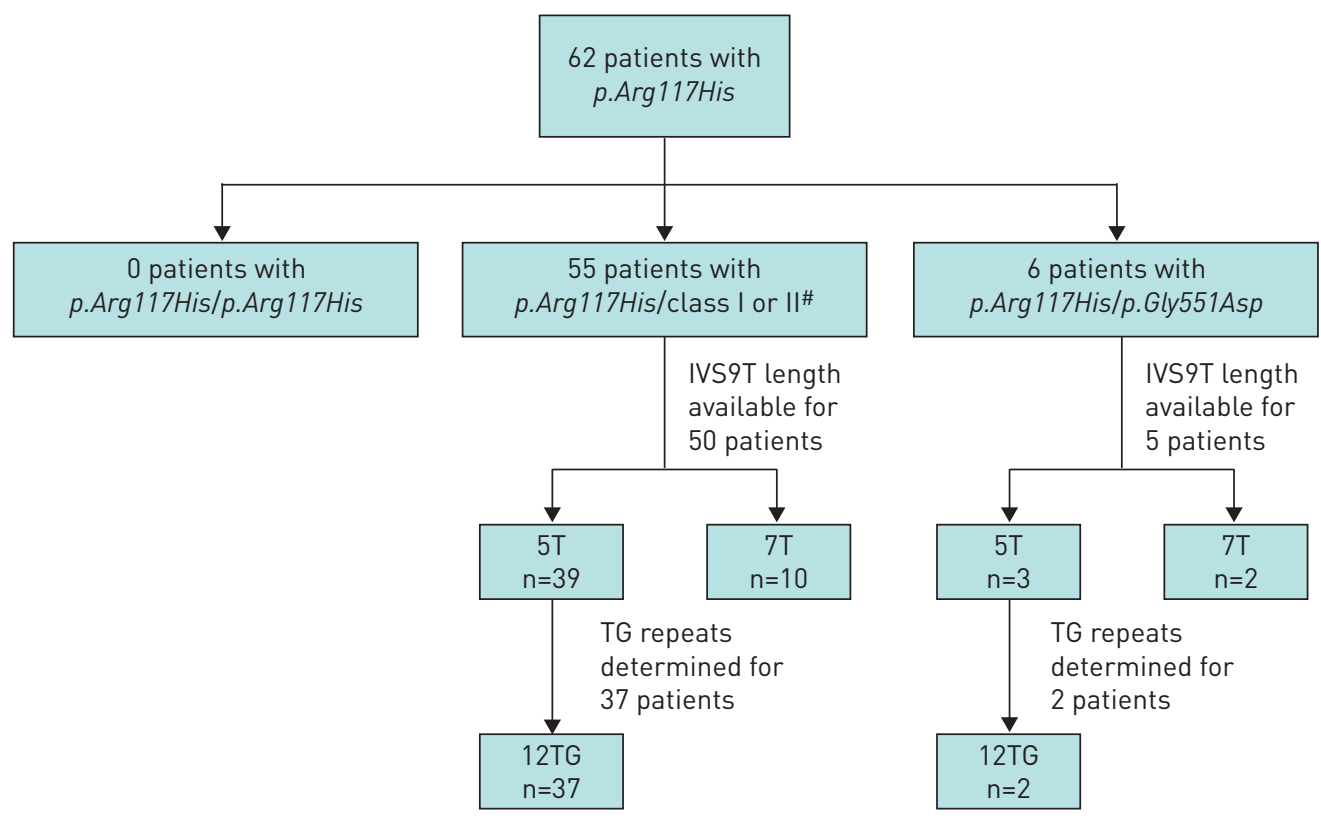

FIGURE 1 Screening of cystic fibrosis database. Patients with p.Arg117His and different polythymidine tract variable sequence on intron 9 (IVS9T) length and thymidine-guanine (TG) repeats. ${ }^{\#}:$ p.Phe508del/p.Arg117His $(\mathrm{n}=49)$, p.Arg560Thr/p.Arg117His (n=3), p.Gly542X/p.Arg117His (n=2) and p.Glu60X/p.Arg117His (n=1). 
p.Arg117His-5T/class I or II $-0.6 \%$, p.Arg117His-7T/class I or II $+0.17 \%$, p.Arg117His/p.Gly551Asp $-0.26 \%$ and p.Phe508del homozygotes $-1.02 \%$. From the model it is estimated that p.Phe508del homozygotes reach an FEV1 \% pred of $50 \%$ at age 36 years, while p.Arg117His-5T/class I or II patients reach an FEV $1 \%$ pred of $50 \%$ at age 67 years. Patients with p.Arg117His-7T/class I or II and p.Arg117His/ p.Gly551Asp would maintain an FEV1 \% pred of $>50 \%$ throughout their lifetime.

Significant differences in the rate of FEV1 decline were found between 5T and p.Arg117His/p.Gly551Asp ( $\mathrm{p}=0.03$ ), and between p.Arg117His/p.Gly551Asp and p.Phe508del homozygotes ( $\mathrm{p}=0.004)$ (table 1). Comparisons between other groups of patients did not reach statistical significance.

\section{Presentation and diagnosis}

We compared the different modes of presentation between the four groups of patients, with Bonferroni corrections for multiple comparisons. The mean $\pm \mathrm{SD}$ age at diagnosis for the different groups was: p.Arg117His-5T 16 \pm 21 years, p.Arg117His-7T 3.4 \pm 10.6 years, p.Arg117His/p.Gly551Asp $13.6 \pm 19$ years and p.Phe508del $1.7 \pm 5.4$ years $(\mathrm{p}<0.0001)$. For patients diagnosed following a symptomatic clinical presentation (rather than newborn screening), the mean \pm SD age at diagnosis was: p.Arg117His-5T 23.9 \pm 24 (median 18) years, p.Arg117His/p.Gly551Asp 22.4 21 (median 25) years and p.Phe508del 1.8 \pm 5.2 (median 0.3) years. Sweat chloride values were significantly different between the four groups, with the following mean $\pm S D$ values: p.Arg117His-5T $82 \pm 14 \mathrm{mEq} \cdot \mathrm{L}^{-1}, \quad$ p.Arg117His-7T $35 \pm 8 \mathrm{mEq} \cdot \mathrm{L}^{-1}, \quad$ p.Arg117His/p.Gly551Asp $83 \pm 38 \mathrm{mEq} \cdot \mathrm{L}^{-1}$ and p.Phe508del $110 \pm 15 \mathrm{mEq} \cdot \mathrm{L}^{-1}$ ( $\mathrm{p}<0.0001$ for all comparisons). 14 (35.9\%) of the p.Arg117His-5T patients were diagnosed following neonatal screening or CF in a sibling, while 18 were diagnosed due to CF-related symptoms: respiratory symptoms (15 patients (38.5\%)), failure to thrive or steatorrhoea (two patients (5.1\%)), meconium ileus (two patients $(5.1 \%)$ ) or infertility (one patient $(2.6 \%)$, some patients had more than one presenting symptom). All nine patients with p.Arg117His-7T for whom information regarding diagnosis was available were diagnosed following neonatal screening (table 2).

\section{Metabolic complications}

$32.4 \%$ of the p.Arg117His-5T patients were pancreatic insufficient, requiring pancreatic enzyme replacement therapy, compared with $11 \%$ of the p.Arg117His-7T patients, $50 \%$ of the p.Arg117His/ p.Gly551Asp patients and $100 \%$ of p.Phe508del homozygotes $(\mathrm{p}<0.001)$. No patients with p.Arg117His had CF-related diabetes versus $11.5 \%$ of p.Phe508del homozygotes ( $\mathrm{p}=0.09)$. One patient (2.7\%) with p.Arg117His-5T had liver disease versus $20.8 \%$ of p.Phe508del homozygotes $(\mathrm{p}=0.02)$. Mean body mass index (BMI) values are shown in table 2 and were lowest for p.Phe508del homozygotes, higher among p.Arg117His/class I or II (with no difference between 5T and 7T) and highest among p.Arg117His/ p.Gly551Asp $(\mathrm{p}<0.0001)$.

\section{Microbiology}

Chronic $P$ aeruginosa colonisation (defined as $>50 \%$ positive cultures per year) was highest among p.Phe508del homozygotes (52\%) and lower in patients with p.Arg117His/class I or II and 5T (26.5\%), with no Pseudomonas colonisation among p.Arg117His/p.Gly551Asp or among patients with p.Arg117His/class I

FIGURE 2 Forced expiratory volume in $1 \mathrm{~s}\left(\mathrm{FEV}_{1}\right) \%$ pred values taken as median values of all recordings over a 6-month period for each patient and the FEV1 per year decline calculated for each of the four groups as shown.

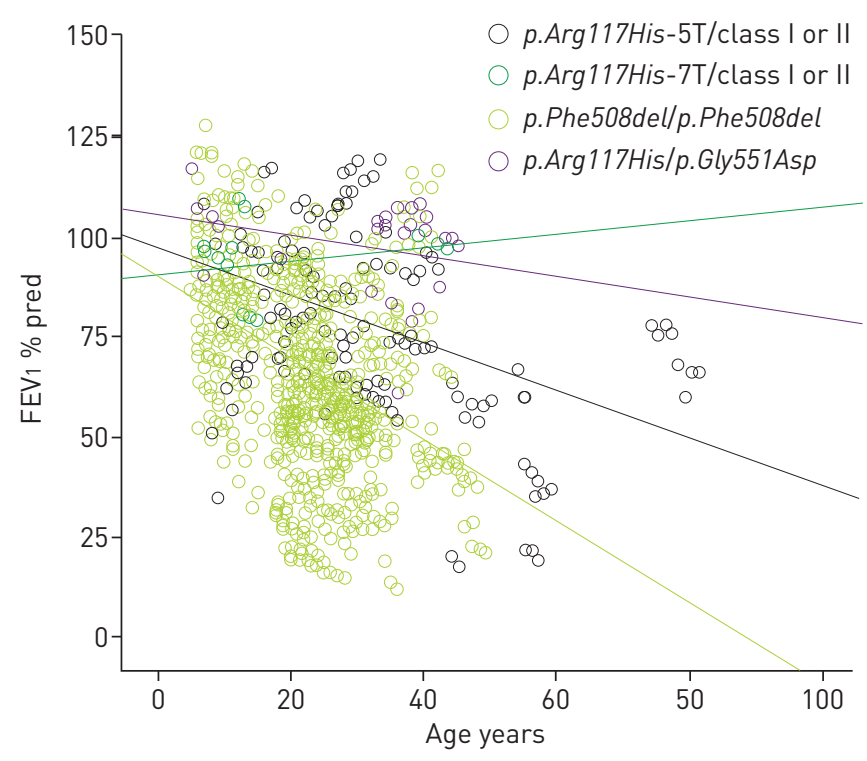




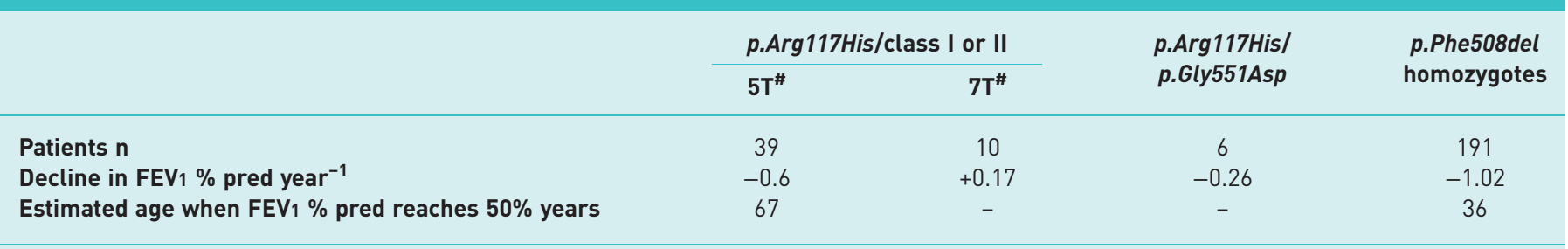

FEV1: forced expiratory volume in $1 \mathrm{~s}$. FEV $1 \%$ pred decline as estimated from the median FEV 1 values for patients in each of the four groups.

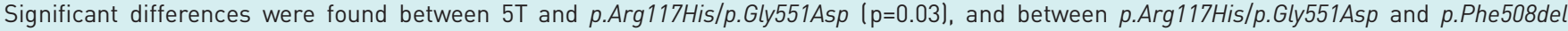
homozygotes $(p=0.004)$. Comparisons between other groups of patients did not reach statistical significance.

or II and $5 \mathrm{~T}(\mathrm{p}<0.0001$ for comparison). As age was different among the groups, this difference may result from acquired Pseudomonas colonisation at older age. To address this, we performed logistic regression for the risk of Pseudomonas colonisation. Homozygosity for p.Phe508del was found to be associated with a risk of 13.4 (95\% CI 4.8-37.7) compared with patients with p.Arg117His-5T with no contribution of age. No differences were noted in prevalence of infection with Staphylococcus aureus (methicillin sensitive or methicillin resistant), Haemophilus influenzae, Stenotrophomonas maltophilia, Burkholderia cepacia complex, Achromobacter or Aspergillus species (table 3).

\section{Discussion}

The clinical severity of CF is largely determined by the class of the CFTR mutation [22]. Mutations that are associated with residual or partial CFTR function are typically associated with a milder phenotype. The p.Arg117His mutation is associated with a poly-T tract and TG repeats which determine the CFTR function. This results in variable clinical consequences. Some patients with p.Arg117His can have significant lung disease, while others have minimal or absent disease and are not diagnosed, as estimated by the finding of reduced prevalence [7].

We found that the rate of lung function decline by age is different between four groups of patients: highest for p.Phe508del homozygotes, lowest for p.Arg117His-7T, and intermediate for p.Arg117His-5T and p.Arg117His/

\section{TABLE 2 Clinical characteristics of p.Arg117His patients}

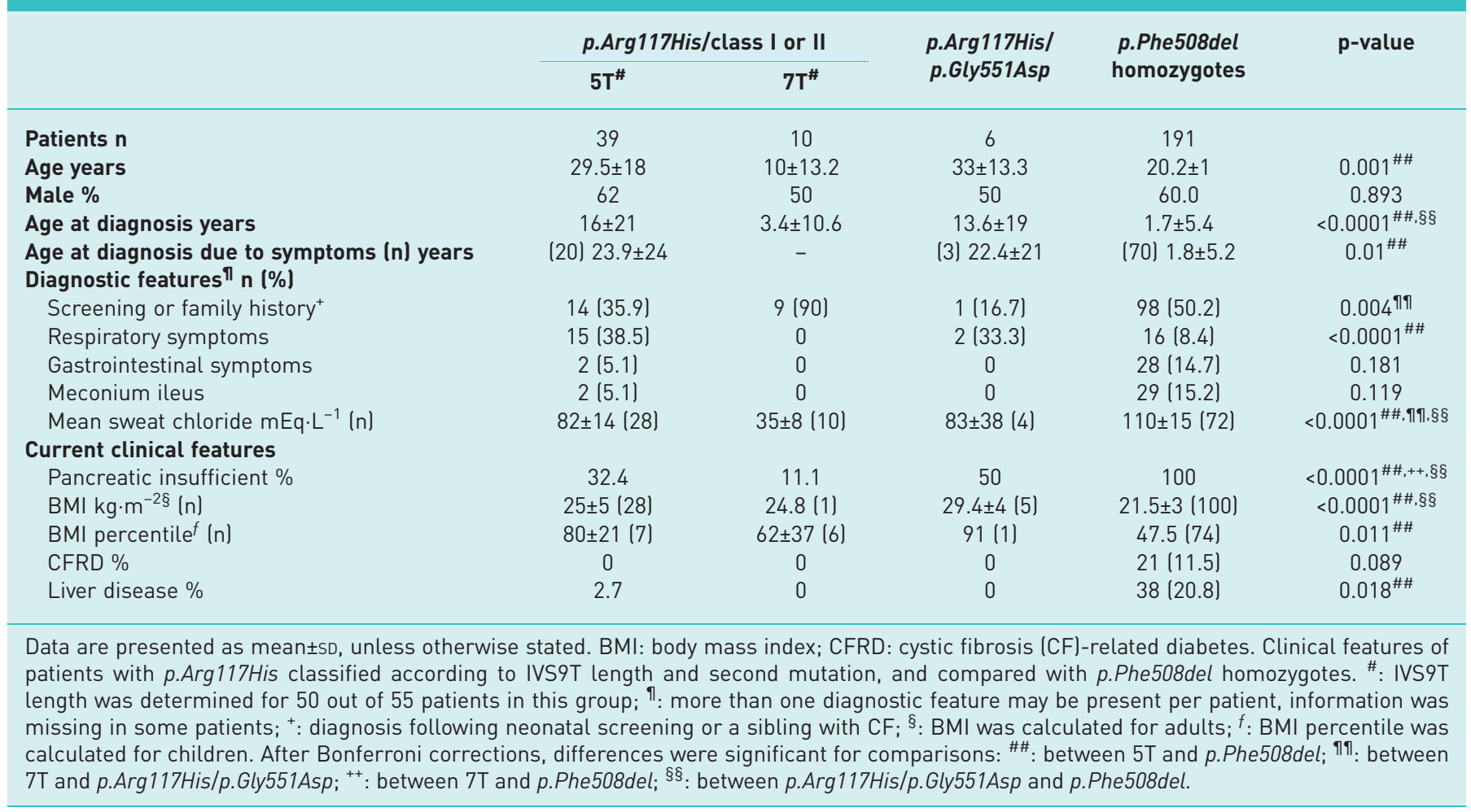


p.Gly551Asp compound heterozygotes, with significant differences in rates of lung function decline between patients with p.Arg117His/p.Gly551Asp and both 5T and p.Phe508del homozygotes. The prediction of the "increase" in lung function among patients with p.Arg117His-7T may result from the young age of the patients and inaccurate extrapolation of the change in FEV1 to older ages. The patients with p.Arg117His/ p.Gly551Asp had either 5T or 7T (figure 1), so the intermediate phenotype may reflect the average of these two subgroups. This is the first time that the rate of lung function decline by age has been shown to be affected among individuals with p.Arg117His and different genetic backgrounds.

We determined IVS9T length by PCR among patients with p.Arg117His. All of these patients were compound heterozygotes for a severe mutation and p.Arg117His. In most of them (50 out of 56) the second mutation was p.Phe508del. We did not check patients' parents for carriage of IVS9 variations and therefore cannot be certain that the "5T" is in cis with the p.Arg117His. However, it has been previously shown that p.Arg117His is in linkage disequilibrium with 5T, whereas the p.Phe508del alleles are always on a 9T background [23]. We can therefore assume that when a poly- $\mathrm{T}$ is found, a $5 \mathrm{~T}$ sequence is in cis with p.Arg117His while the longer sequence is associated with the second mutation, at least in the 50 patients with p.Phe508del/p.Arg117His. Among patients with p.Arg117His, the most severe phenotype was associated with an IVS9T length of 5 and a class I/II mutation, followed by an IVS9T length of 7 and a class I/II mutation. A small group of patients with p.Arg117His and p.Gly551Asp had features with inconsistent severity (higher sweat chloride and pancreatic insufficiency but no Pseudomonas colonisation and a higher BMI). All patients in whom TG repeats could be determined had 12 TG repeats; therefore, a further classification according to TG repeat number could not be performed. A sequence of 12 TG repeats may be in linkage disequilibrium with the p.Arg117His mutation or with an IVS9T length of 5 in our population.

Interestingly, we found fewer patients with p.Arg117His-7T than with -5T. This could be explained by reduced penetrance of the p.Arg117His mutation, as was hypothesised by THAuvin-RoBINET et al. [7]. Indeed, higher detection through newborn screening (introduced in 1983) may explain the younger average age and age at diagnosis of the patients with p.Arg117His-7T, assuming that most of these patients were too mild to have been diagnosed because of symptoms. We found no patients homozygous for p.Arg117His or compound heterozygous for p.Arg117His and a class IV/V mutation. These classes of mutation are rare, and individuals carrying these combinations of mutations who may either be healthy, have CFTR-related metabolic syndrome or have a very atypical CF phenotype are not diagnosed with CF $[7,24]$.

However, we have found that 37 patients carrying the p.Arg117His mutation with an IVS9T length of 5 and a second class I or II mutation were quite severely affected. Of these patients, $32 \%$ were pancreatic insufficient and $25 \%$ were chronically colonised with $P$. aeruginosa. Two such patients had meconium ileus at birth; one patient with this genotype was infected with B. cepacia complex and another with methicillin-resistant $S$. aureus. The FEV1 decline was smaller but not significantly different from p.Phe508del homozygotes. This could not be attributed to the older age of p.Phe508del homozygotes and we were not able to find an age at which there was a change in the slope of lung function decline. These findings argue in favour of the need for early detection and treatment of patients with p.Arg117His with an IVS9T length of 5.

TABLE 3 Bacterial isolates in sputum samples from p.Arg117His patients versus p.Phe508del homozygotes

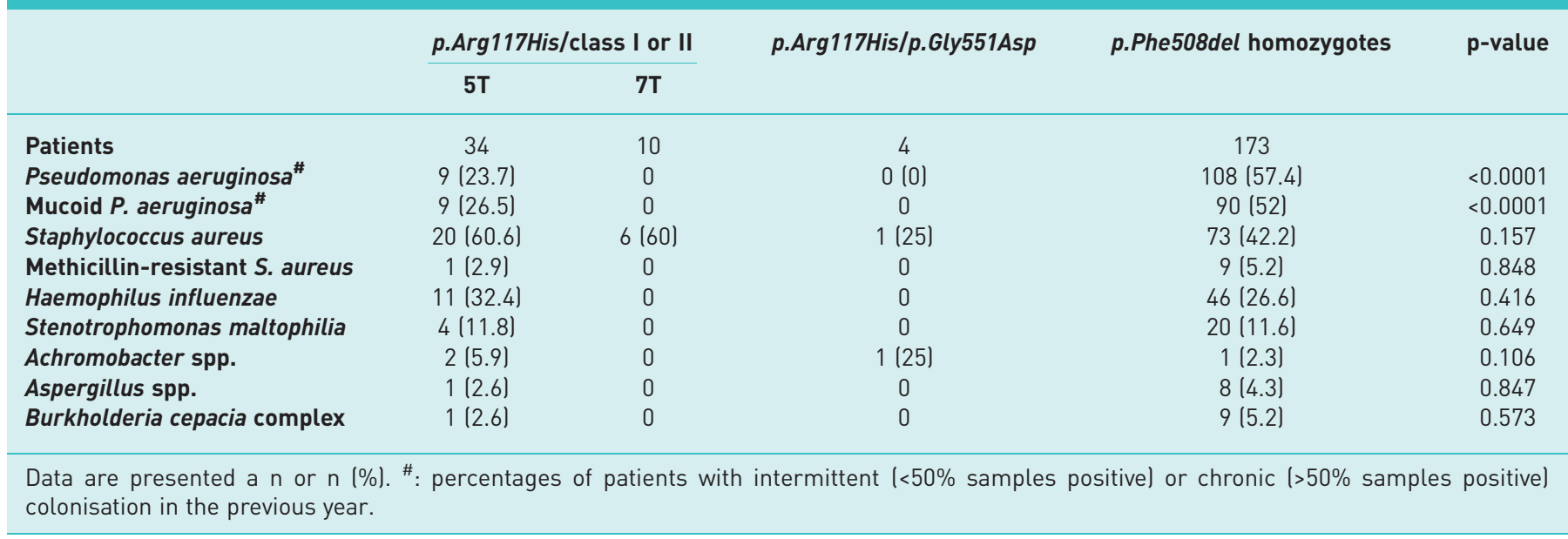


This study was a single-centre study, which limited the number of patients as well as longitudinal lung function data. However, patients in this centre had consistent care and we were able to ascertain full details for all patients. As newborn screening has been available since the 1980s, we have ascertainment of almost all CF cases. Rate of FEV1 decline of p.Phe508del homozygotes was $1.02 \%$ per year, which is comparable to previous studies [25-27].

Some of the differences between the groups may be attributed to the younger age, and age at diagnosis, of $7 \mathrm{~T}$ versus $5 \mathrm{~T}$ patients. However, the differences in sweat chloride values and pancreatic status are typically not influenced by age, and likely represent lower CFTR function in p.Arg117His-5T. In patients with the p.Arg117His mutation, a class I/II mutation in trans and a poly-T sequence of 5 on intron 9 in cis convey a more severe phenotype, which has similar rates of decline in FEV1 compared with p.Phe508del homozygotes. These findings support the inclusion of the p.Arg117His mutation in newborn screening panels and the potential treatment of such patients with a CFTR potentiator.

\section{Acknowledgements}

J.S. Elborn has served as an investigator in Vertex Pharmaceuticals Incorporated clinical studies; has participated in advisory boards or as a consultant for Vertex Pharmaceuticals Incorporated, Novartis, Bayer, Actavis and Boehringer Ingelheim, and has received grant support from Gilead and Novartis.

M. Shteinberg was a visiting research fellow at Queen's University Belfast and received financial support from Novartis Pharmaceuticals.

We acknowledge with gratitude Katherine Lavery, Carina Loughran, Danielle Corey, Joyce Murray, Jane Bell and Jane Carson (Northern Ireland Regional Adult and Paediatric Cystic Fibrosis Centres, Belfast, UK) for assistance with lung function data; Doron Behar (Gene by Gene Ltd, Houston, TX, USA) for assisting with the genetic interpretation; and Elinor Johnston, Susan Martin, Katherine O’Neill and Judy Bradley (Northern Ireland Regional Adult and Paediatric Cystic Fibrosis Centres, Belfast, UK) for assistance with other aspects this project.

\section{References}

Zielenski J, Tsui LC. Cystic fibrosis: genotypic and phenotypic variations. Annu Rev Genet 1995; 29: 777-807.

Kerem E, Kerem B. Genotype-phenotype correlations in cystic fibrosis. Pediatr Pulmonol 1996; 22: 387-395. Sheppard DN, Rich DP, Ostedgaard LS, et al. Mutations in CFTR associated with mild-disease-form $\mathrm{Cl}^{-}$channels with altered pore properties. Nature 1993; 362: 160-164.

4 Bienvenu T, Beldjord C, Adjiman M, et al. Male infertility as the only presenting sign of cystic fibrosis when homozygous for the mild mutation R117H. J Med Genet 1993; 30: 797.

5 Gervais R, Dumur V, Rigot JM, et al. High frequency of the R117H cystic fibrosis mutation in patients with congenital absence of the vas deferens. N Engl J Med 1993; 328: 446-447.

6 de Nooijer RA, Nobel JM, Arets HG, et al. Assessment of CFTR function in homozygous R117H-7T subjects. J Cyst Fibros 2011; 10: 326-332.

7 Thauvin-Robinet C, Munck A, Huet F, et al. The very low penetrance of cystic fibrosis for the R117H mutation: a reappraisal for genetic counselling and newborn screening. J Med Genet 2009; 46: 752-758.

8 Scotet V, Audrezet MP, Roussey M, et al. Immunoreactive trypsin/DNA newborn screening for cystic fibrosis: should the R117H variant be included in CFTR mutation panels? Pediatrics 2006; 118: e1523-e1529.

9 Comer DM, Ennis M, McDowell C, et al. Clinical phenotype of cystic fibrosis patients with the G551D mutation. QJM 2009; 102: 793-798.

10 Chu CS, Trapnell BC, Curristin S, et al. Genetic basis of variable exon 9 skipping in cystic fibrosis transmembrane conductance regulator mRNA. Nat Genet 1993; 3: 151-156.

11 Chillón M, Casals T, Mercier B, et al. Mutations in the cystic fibrosis gene in patients with congenital absence of the vas deferens. N Engl J Med 1995; 332: 1475-1480.

12 Anguiano A, Oates RD, Amos JA, et al. Congenital bilateral absence of the vas deferens: a primarily genital form of cystic fibrosis. JAMA 1992; 267: 1794-1797.

13 Cottin V, Thibout Y, Bey-Omar F, et al. Late CF caused by homozygous IVS8-5T CFTR polymorphism. Thorax 2005; 60: 974-975

14 Van Hoorenbeeck K, Storm K, van den Ende J, et al. N1303K and IVS8-5T, clinical presentation within a family with atypical cystic fibrosis. J Cyst Fibros 2007; 6: 220-222.

15 Noone PG, Pue CA, Zhou Z, et al. Lung disease associated with the IVS8 5T allele of the CFTR gene. Am J Respir Crit Care Med 2000; 162: 1919-1924.

16 Massie RJ, Poplawski N, Wilcken B, et al. Intron-8 polythymidine sequence in Australasian individuals with CF mutations R117H and R117C. Eur Respir J 2001; 17: 1195-1200.

17 Zuccato E, Buratti E, Stuani C, et al. An intronic polypyrimidine-rich element downstream of the donor site modulates cystic fibrosis transmembrane conductance regulator exon 9 alternative splicing. J Biol Chem 2004; 279: 16980-16988.

18 Pagani F, Buratti E, Stuani C, et al. Splicing factors induce cystic fibrosis transmembrane regulator exon 9 skipping through a nonevolutionary conserved intronic element. J Biol Chem 2000; 275: 21041-21047.

19 Niksic M, Romano M, Buratti E, et al. Functional analysis of cis-acting elements regulating the alternative splicing of human CFTR exon 9. Hum Mol Genet 1999; 8: 2339-2349.

20 Groman JD, Hefferon TW, Casals T, et al. Variation in a repeat sequence determines whether a common variant of the cystic fibrosis transmembrane conductance regulator gene is pathogenic or benign. Am J Hum Genet 2004; 74: $176-179$.

21 Mantovani V, Garagnani P, Selva P, et al. Simple method for haplotyping the poly(TG) repeat in individuals carrying the IVS8 5T allele in the CFTR gene. Clin Chem 2007; 53: 531-533. 
22 Sosnay PR, Siklosi KR, Van Goor F, et al. Defining the disease liability of variants in the cystic fibrosis transmembrane conductance regulator gene. Nat Genet 2013; 45: 1160-1167.

23 Cordovado SK, Hendrix M, Greene CN, et al. CFTR mutation analysis and haplotype associations in CF patients. Mol Genet Metab 2012; 105: 249-254.

24 Ren CL, Desai H, Platt M, et al. Clinical outcomes in infants with cystic fibrosis transmembrane conductance regulator (CFTR) related metabolic syndrome. Pediatr Pulmonol 2011; 46: 1079-1084.

25 Davis PB, Byard PJ, Konstan MW. Identifying treatments that halt progression of pulmonary disease in cystic fibrosis. Pediatr Res 1997; 41: 161-165.

26 Schaedel C, De Monestrol I, Hjelte L, et al. Predictors of deterioration of lung function in cystic fibrosis. Pediatr Pulmonol 2002; 33: 483-491.

27 Taylor-Robinson D, Whitehead M, Diderichsen F, et al. Understanding the natural progression in \%FEV1 decline in patients with cystic fibrosis: a longitudinal study. Thorax 2012; 67: 860-866. 